\title{
CAMBIOS EN LA POSICIÓN DE MERCADO DE LAS EMPRESAS COLOMBIANAS*
}

\author{
Andrés Ramírez Hassan** \\ Santiago García Peláez $z^{* \star *}$ \\ José David Garcés Ceballos ${ }^{\star \star * \star}$ \\ Recibido: marzo 25 de 2011 \\ Aceptado: septiembre 30 de 2011
}

\section{RESUMEN}

En este trabajo se presentan los resultados de una investigación empírica sobre los cambios en la posición de mercado de las empresas colombianas, según la matriz crecimiento-participación desarrollada por la firma Boston Consulting Group. Para ello se utilizan datos de la Superintendencia de Sociedades de Colombia y se aplican modelos logit multinomiales. Los resultados indican que en términos generales, las variables financieras afectan de manera similar la posición de mercado de todas las empresas, mientras que variables de organización industrial tienen efectos distintos que dependen del tipo de empresa. Las conclusiones del trabajo sugieren que hay amplio campo de acción para los responsables de política con el fin de estimular el éxito empresarial.

\section{PALABRAS CLAVE}

Matriz crecimiento-participación, restricciones financieras, concentración de mercado, poder de mercado.

\section{CLASIFICACIÓN JEL} L22; L25.

\section{CONTENIDO}

Introducción; 1. Marco de referencia; 2. Datos; 3. Metodología; 4. Modelo y resultados; 5. Conclusiones; Bibliografía; Anexos.

\footnotetext{
Este artículo de investigación surge como producto asociado a un interrogante general que se analiza en el grupo investigación de Economía y Empresa de la Universidad EAFIT, ¿Qué explica el ciclo de vida de las empresas colombianas? El grupo de investigación en consideración está clasificado en categoría B por COLCIENCIAS, y el artículo en cuestión es financiado por la Universidad EAFIT, Medellín, Colombia. La vigencia del proyecto es el año 2011.

** Estudiante del Doctorado en Ciencia-Estadística, Universidad Nacional de Colombia, Medellín, Colombia. Máster en Finanzas, Universidad EAFIT, Medellín, Colombia. Máster en Economía, Universidad Nacional de Colombia, Medellín, Colombia. Economista, Universidad Nacional de Colombia, Medellín, Colombia. Docente e Investigador, Universidad EAFIT, Medellín, Colombia. Miembro activo del grupo de investigación de Economía y Empresa. Carrera 36B \#40AA SUR 116, Envigado (Colombia). Teléfono +57(4) 3324228. Correo electrónico: aramir21@eafit.edu.co.

*** Estudiante de último semestre de Economía de la Universidad EAFIT, Medellín, Colombia. Carrera 13B \#4B Sur 205, Medellín, Colombia. Correo electrónico: sgarcia5@eafit.edu.co.

**** Estudiante de último semestre de economía de la Universidad EAFIT, Medellín, Colombia. Integrante del grupo de investigación en Economía y Empresa. Calle 20 sur \#41AA-161, Medellín, Colombia. Teléfono +57(4) 3223069. Correo electrónico: jgarces3@eafit. edu.co.
} 


\section{CHANGES IN MARKET POSITION OF COLOMBIAN COMPANIES}

\section{ABSTRACT}

This paper presents the results of an empirical research on changes in market position that Colombian companies, according to the growth-share matrix developed by the Boston Consulting Group. For this purpose, data from the Colombian Society Regulation Office are used applied to multinomial logit models. The results indicate that, in general terms the financial variables affect similarly the market position of all companies, while the industrial organization variables have different effects that depend on the type of company. The conclusions of this paper suggest that there is a broad field of action for policy makers with the end of stimulating business success.

\section{KEY WORDS}

Growth-share matrix, financial constrains; market concentration; market power

\section{JEL CLASSIFICATION}

L22, L25

\section{CONTENT}

Introduction; 1. Reference framework; 2. Data; Methodology; Model and results; 5. Conclusions; Bibliography; Attachments.

\section{MUDANÇAS NA POSIÇÃO DE MERCADO DAS EMPRESAS COLOMBIANAS}

\section{RESUMO}

Este artigo apresenta os resultados de uma pesquisa empírica sobre as mudanças na posição de mercado das empresas colombianas, de acordo com a matriz de crescimento-participação desenvolvida pela firma Boston Consulting Group. Para este proposito, dados da Superintendência de Sociedades Colombianas são usados e modelos logit multinominais são aplicados. Os resultados indicam que, em geral, as variáveis financeiras afetam igualmente a posição de mercado de todas as empresas, enquanto que as variáveis da organização industrial têm efeitos diferentes dependendo do tipo de empresa. As conclusões do estudo sugerem os decisores políticos tem uma ampla margem para estimular o sucesso do negocio.

\section{PALAVRAS-CHAVE}

Crescimento da matriz crescimento-participação, restrições financeiras, a concentração de mercado, poder de mercado.

\section{CLASSIFICAÇÃO JEL} L22, L25.

\section{CONTEÚDO}

Introdução: 1. Quadro 2. Dados 3. Metodologia, 4. Modelo e os resultados 5. Conclusões, Bibliografia; 


\section{INTRODUCCIÓN}

El estudio de casos de éxito empresarial es un tema popular en las universidades con escuelas de negocios y economía; sin embargo, tan importante como estudiar los casos de éxito, es igualmente relevante conocer los casos de fracaso empresarial. Es así que para analizar los factores determinantes del éxito o fracaso de las empresas colombianas, se toma como referencia el cambio en la posición de mercado establecido a partir de la caracterización del Boston Consulting Group (BCG). Esta metodología clasifica a las empresas en cuatro categorías: empresas Vaca, empresas Estrella, empresas Interrogante y empresas Perro, donde la definición de las categorías está estrechamente ligada a la participación de las ventas de las empresas en su respectivo sector económico, y al crecimiento de los ingresos de las mismas en un determinado horizonte temporal. En consecuencia, la definición de cambio en la posición de mercado está asociada al cambio en la categoría de una empresa en cierto horizonte temporal.

Aunque es difícil modelar las causas que llevan a las empresas a evidenciar cambios en la posición de mercado debido a que estas causas pueden ser no observables o puramente accidentales, es importante examinar la teoría de las finanzas corporativas y de la organización industrial para encontrar factores en común que compartan las empresas perdedoras o ganadoras. Es así que la identificación de factores comunes que explican el cambio de la posición de mercado puede ayudar a la formulación de políticas microeconómicas y macroeconómicas.

El análisis a desarrollar adopta un enfoque dual, de carácter financiero y microeconómico. Esto ofrece ciertas ventajas por dos motivos: primero, puede servir de guía para la toma de decisiones empresariales en la firma; y segundo, puede facilitar la comprensión de las estructuras de mercado en la economía colombiana y la forma en que estas estructuras inciden en el desempeño de las empresas. Específicamente, el enfoque permite tener en cuenta factores de carácter financiero asociados exclusivamente a cada empresa y factores de mercado comunes entre estas, según la industria donde las mismas operen. El análisis de tipo financiero se concentrará en dos áreas fundamentales: liquidez y rentabilidad. La liquidez es importante para que una empresa pueda sobrevivir a desbalances temporales o cambios inesperados en el mercado, y pueda tener capacidad de adelantar procesos de expansión. Por su parte, la rentabilidad es el elemento fundamental que asegura la supervivencia de la empresa en el largo plazo, pues al obtener ganancias marginales superiores a la inversión requerida por las empresas se genera valor agregado.

El análisis microeconómico lo provee la teoría de la organización industrial. Los factores de mercado son muy importantes, pues son las mismas estructuras de mercado las que en algunos casos pueden generar problemas de liquidez o rentabilidad para algunas empresas. Así, por ejemplo, en sectores donde el poder de los clientes es alto, como el caso de los proveedores de almacenes de cadena, la presión que los grandes clientes generan sobre los pequeños productores resulta en mantenimiento de altos niveles de inventarios y cartera por parte de los últimos, lo que provoca dificultades de liquidez. Igualmente, en sectores poco rentables por la penetración de productos internacionales más competitivos, como el calzado o los textiles, empresas que operan permanentemente con rentabilidades bajas o negativas experimentan una disminución de su valor. Bajo esta concepción, se estudiarán como factores relacionados con la estructura de mercado, los niveles de barreras de entrada, el grado de concentración del mercado y las externalidades por efectos de aglomeración que inciden en la probabilidad de un cambio en la posición de mercado de las empresas colombianas.

El objetivo de este trabajo es investigar empíricamente qué factores inciden en el cambio de 
posición de mercado de las empresas colombianas. Específicamente, determinar si existen variables comunes a las empresas que afecten la probabilidad de cambio en la posición de mercado. La base de datos utilizada proviene de la Superintendencia de Sociedades de Colombia (SSC) y comprende los estados financieros de empresas no financieras entre 1995 y 2009. La metodología econométrica utilizada son los modelos logit multinomiales, y los principales resultados indican que la mayoría de las empresas se benefician de una alta generación de flujo de caja y de ser eficientes en la generación de efectivo. Adicionalmente, se encuentra que las empresas con más años de permanencia en el mercado son bastante estables, comparadas con las empresas jóvenes, las cuales experimentan una alta probabilidad de cambio de posición en el mercado, bien sea positiva o negativa. Al respecto de la estructura de mercado, las barreras de entrada y el tamaño de las empresas tienen efectos completamente diferentes según el tipo de empresa analizada; algo similar ocurre con respecto al tamaño de las firmas. También se encuentra que, en general, las empresas ubicadas en los mercados más concentrados son las que evidencian menor probabilidad de cambio.

Este trabajo contiene seis secciones: esta introducción es la primera; la segunda sección contiene el marco de referencia, en el que se hace un recuento sobre la matriz crecimiento-participación y una revisión de la literatura teórica y empírica; la tercera sección contiene una descripción de los datos y comenta su construcción; la cuarta sección contiene la metodología utilizada; en la quinta sección se presentan los resultados y finalmente, se concluye.

\section{MARCO DE REFERENCIA}

\subsection{La matriz de crecimiento-participación}

A finales de 1960, la firma consultora americana Boston Consulting Group (BCG) desarrolló un método simple para visualizar de forma compacta las ventajas y desventajas de una unidad de negocio; este método de análisis se denominó matriz crecimiento-participación y cartera de productos. En su esencia, el producto desarrollado por BCG es un modelo gerencial estructurado sobre un marco de análisis de tipo microeconómico. Al respecto, Hax y Maljuf (1984) identifican que en este enfoque la firma se presenta en términos de un portafolio o una cartera de diversos productos; cada uno ofrece diferentes niveles de crecimiento y rentabilidad. Cada producto o unidad de negocio es ubicado en un plano separado en cuadrantes, en donde el eje horizontal representa la participación de mercado y el eje vertical representa el crecimiento de la industria a la cual pertenece la firma. Mientras que el eje vertical es incremental a medida que hay una mayor distancia respecto al origen, el eje horizontal es incremental a medida que se reduce la distancia respecto a este. En la figura 1 se encuentra una descripción gráfica de la matriz. A continuación se hace una descripción de los cuatro tipos de empresas que resultan de la caracterización según la matriz del BCG.

Empresas Perro: las empresas ubicadas en el cuadrante uno son denominadas empresas tipo Perro y se caracterizan por tener una generación moderada de flujo de caja, dado que tienen una participación de mercado baja en un sector de bajo crecimiento. Estas firmas son las menos atractivas, pues el poco flujo de caja que generan es absorbido nuevamente por la operación de la empresa.

Empresas Interrogante: las empresas ubicadas en el cuadrante dos son llamadas empresas tipo Interrogante y por lo general generan flujos de caja cercanos a cero o negativos. Dado que tienen una cuota de mercado pequeña, en un sector de alto crecimiento, se enfrentan a la necesidad de reinvertir gran parte del efectivo 
que genera la operación. Según las fortalezas y decisiones estratégicas de estas firmas, una vez el mercado madura pueden convertirse en empresas perdedoras (Empresas Perro) o ganadoras (Empresas Estrella o Vacas Lecheras).

Empresas Estrella: las empresas ubicadas en el cuadrante tres son llamadas empresas tipo Estrella y por lo general presentan flujos de caja positivos, aunque no muy altos. Dado que tienen una posición de mercado exitosa en un contexto de alto crecimiento generan un alto volumen de ingresos, pero una alta proporción es requerida para mantener el liderazgo relativo en el mercado. En el momento en que el mercado madure, estas empresas generalmente se convierten en Empresas Vaca.

Empresas Vaca: las empresas ubicadas en el cuadrante cuatro son llamadas empresas tipo Vaca y por lo general reportan flujos de caja considerablemente positivos. Al tener una alta participación en un mercado maduro de poco crecimiento, estas empresas generan un flujo de caja superior al que es necesario reinvertir, por lo que tienen excedentes de recursos que pueden ser invertidos en empresas Estrella o empresas Interrogante.

Figura 1. Matriz crecimiento-participación

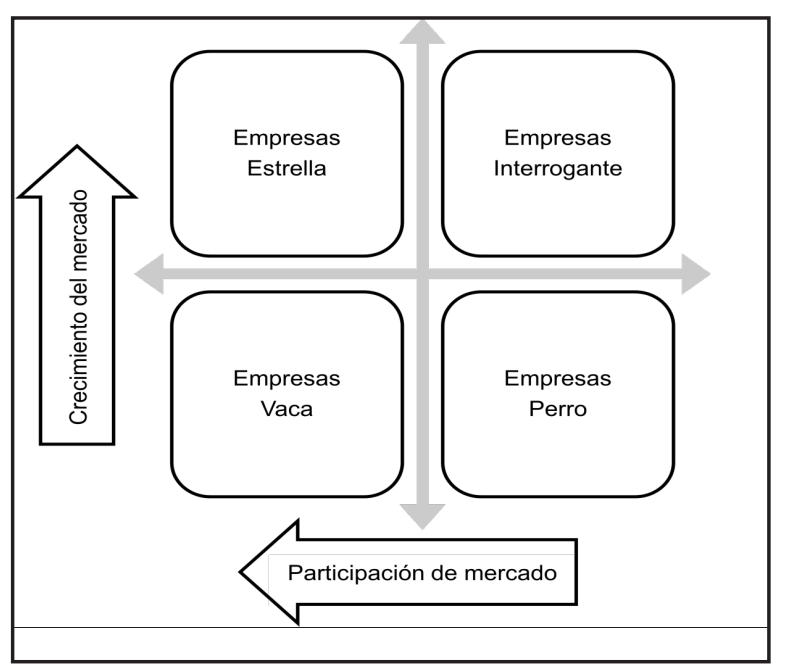

Fuente: Elaboración propia.

\subsection{Finanzas corporativas y organización industrial}

El cambio de posición de una firma está explicado por la teoría del ciclo de vida de las empresas. Para estas, la toma de decisiones, es decir, la estrategia empresarial, es de vital importancia para generar ventajas competitivas en su mercado y sobre los competidores. Estas decisiones dependen de la posición de mercado en la cual se encuentren las firmas. Así, según Cardona y Cano (2004), empresas que buscan entrar a una industria deben abrirse paso a través de las barreras de entrada que se les presenten (situación propia de las empresas Interrogante); luego, con el paso del tiempo y la introducción de nuevos procesos y mejores relaciones en el mercado, las empresas comienzan a obtener más ganancias, aunque los costos sean elevados (como las empresas Estrella); las empresas que se consolidan por la experiencia adquirida, acumulación de capital de trabajo, etc. buscan mantener esa posición (como las empresas Vaca); finalmente, empresas con bajo flujo de caja debido a exceso de capacidad instalada, disminución de utilidades y clientes, son empresas poco atractivas y con tendencia a salir del mercado (como es más común en las empresas Perro).

Dado lo anterior, se pueden identificar algunos conceptos básicos para entender estas situaciones. En principio, la ley de Gibrat ${ }^{1}$ establece que el tamaño de la empresa y su crecimiento están positivamente relacionados. No obstante, Bechetti y Trovato (2001) encontraron en su estudio, en el que pretendían probar las limitaciones de esta ley, que no sólo el tamaño y la edad de la empresa explican su crecimiento, sino también factores de mercado y de financiación.

El crecimiento de una empresa en un período determinado es dependiente de su tamaño en el período anterior. Esto implica que tanto la edad como el tamaño son variables que explican cambios en el crecimiento de una empresa, sin tener en cuenta las rentas del crecimiento del mercado para esta y el crecimiento de la industria en sí. Para más detalles y evidencias sobre la ley de Gibrat consultar Bachetti y Trovato (2001). 
A estas variables se les suman también la situación espacial de las firmas y las barreras de entrada que estas enfrentan. En este sentido, Aláez, Longás y Ullibarri (2001) encuentran que la ubicación de las firmas en un determinado espacio obedece a las necesidades de infraestructura y recursos que les permitan un mayor desarrollo, y muestran que empresas con una mayor necesidad de tecnología se encuentran más aglomeradas en las ciudades, por el contrario cuando desciende el nivel de exigencia tecnológica, las firmas se ven favorecidas por su ubicación en lugares más alejados de las ciudades, donde los recursos básicos sean más baratos.

Por su parte, Cardona y Cano (2004, p. 15) encuentran, respecto a las barreras de entrada, que:

Como parte del ciclo, es posible que con el tiempo la ventaja de costos de las empresas ya establecidas se amplíe, hasta el punto en que no se presenten nuevas entradas suficientemente innovadoras como para garantizar la permanencia de nuevas empresas en el mercado. Los costos fijos generan una estructura de mercado de competencia imperfecta, en tanto que restringen la entrada. Asimismo, la apropiada acumulación de capital reduce los costos de producción y se acentúa con el proceso de experiencia, a través de la investigación y la innovación.

Además, para el caso de empresas de menor tamaño (MyPIME), encuentran que variables idiosincráticas de los dueños y su capacidad financiera son las principales barreras.

Del lado de las finanzas, Carpenter y Petersen (2002) encontraron, para una muestra de $1.600 \mathrm{em}$ presas a través de un panel de datos tratado con un modelo de primeras diferencias, que las empresas pequeñas que se financian con la mayoría de sus ingresos tienen un crecimiento similar o mayor al de su flujo de caja y acuden poco a la financiación externa.

Para Colombia no se encontraron ejercicios de este tipo, pero Tenjo, López y Zamudio (2006), en un estudio de la estructura de capital de las empresas colombianas en el período 1996-2002, utilizaron, como regresores, variables financieras de las empresas bajo estudio que permitieran mirar su estructura de capital, con el fin de observar su forma de financiamiento.

Langebaeck (2008) utiliza la base de datos de la Superintendencia de Sociedades para investigar los determinantes micro y meso económicos de los ingresos operacionales de las empresas no financieras en Colombia. Después de practicar un ejercicio econométrico de regresión por cuantiles se desprenden cuatro conclusiones principales: primero, no se cumple la Ley de Gibrat, los resultados muestran que las empresas más pequeñas crecen más rápido que las grandes; segundo, la edad resulta significativa para explicar el tamaño de las empresas, pero los efectos son diferentes según el tamaño de la empresa: en el decil inferior es positivo, mientras que en los deciles más altos es negativo; tercero, el capital extranjero no contribuye a explicar el crecimiento en deciles bajos pero tiene un efecto significativo y positivo en deciles más altos, y cuarto, no se corrobora la hipótesis de que la concentración accionaria es positiva para el crecimiento de las empresas por sus efectos sobre la calidad de la supervisión de las conductas de los administradores.

\section{DATOS}

La base de datos que se utiliza proviene de la Superintendencia de Sociedades de Colombia (SSC). Allí se encuentran disponibles, entre otros documentos, los Balances Generales, Estados de Resultados y Estado de Flujo de Efectivo de todas las empresas que reportan ante la SSC. Con base en la información contenida en estos tres estados financieros se divide a las empresas en los cuatro grupos (ver figura 1), según el enfoque de la cartera de productos; posteriormente, se construyen variables de interés de corte financiero y microeconómico. En esta sección se detalla la forma en que 
se modifica la base de datos; posteriormente, se describen las variables utilizadas y finalmente se presentan estadísticas descriptivas de los datos.

\subsection{Depuración de la base de datos}

La base de datos original es un panel con observaciones anuales sobre las empresas en el período 1995-2009. Sobre esta base de datos se hicieron dos depuraciones fundamentales. En primer lugar, para asegurar la existencia de los sujetos bajo estudio en todo el período, el panel fue balanceado, con la exclusión de las empresas que no cumplieran con esa condición. La finalidad de este procedimiento es asegurar que se tiene información completa sobre todos las empresas en todo el período. Al realizar este procedimiento, la muestra se redujo a 9.448 observaciones.

Segundo, aunque podría trabajarse directamente con la base de datos balanceada, realizar el análisis de la matriz de BCG durante períodos cortos de tiempo (anuales) puede resultar en conclusiones equivocadas, pues en estos pueden ocurrir cambios transitorios en el mercado, que no reflejan las condiciones estructurales como lo señalan Stalk y Stern (2002). Por tanto, se ha optado por trabajar con los promedios de cada empresa asociados a dos períodos: el primero está comprendido entre 1995 y 2002, y el segundo consta del promedio de las observaciones entre 2003 y 2009. El criterio para la selección de estos períodos muestrales es tomar en consideración la mayor cantidad de información disponible, y trabajar con períodos balanceados, puesto que al estimar la tasa de crecimiento necesariamente se pierde la primera observación, es así que cada período consta de 7 años. Adicionalmente, el cálculo del promedio resume la información contenida en todas las realizaciones, y no solo años puntuales. También se eliminaron empresas con observaciones atípicas, y en consecuencia, al realizar esta depuración la muestra se redujo a 3.448 observaciones.
Dado que la matriz crecimiento-participación exige tener una distinción clara entre empresas con una participación de mercado alta y baja, y entre sectores con crecimiento alto y bajo, fue necesario fijar los criterios para definir estos rangos. Se identificó el tipo de empresa según los siguientes criterios (ver tabla 1):

- Participación de Mercado: se definió como el nivel de ventas de la empresa en relación con el nivel de ventas de la empresa líder del sector al que pertenece. Se calificó a las empresas que tuvieron una participación relativa menor o igual al 25\% de las ventas de la empresa líder como empresas "Perro" o "Interrogante", y aquellas con participación mayor a ese valor, son empresas "Estrella" y "Vaca". Bruce Henderson (1976), citado por en Stalk y Stern (2002, p. 250), sostiene que una participación baja de mercado se define en el momento en que la cuota de mercado de un competidor no alcanza el 25\% de la cuota de mercado del competidor líder. Aunque esta afirmación no es una regla que haya sido sometida a pruebas rigurosas, es una hipótesis que parece ajustarse bastante bien a los datos.

Tabla 1. Criterios de identificación de las empresas

\begin{tabular}{|c|c|c|}
\hline $\begin{array}{l}\text { Tipo de } \\
\text { empresa }\end{array}$ & $\begin{array}{l}\text { Participación } \\
\text { Relativa }\end{array}$ & $\begin{array}{l}\text { Crecimiento } \\
\text { del Sector }\end{array}$ \\
\hline Perro & $\begin{array}{l}\text { Menor o igual } \\
\text { al } 25 \%\end{array}$ & $\begin{array}{l}\text { Crec. ventas sector } \\
<1.5^{*} \text { Crec. ventas país }\end{array}$ \\
\hline Interrogante & $\begin{array}{l}\text { Menor o igual } \\
\text { al } 25 \%\end{array}$ & $\begin{array}{l}\text { Crec. ventas sector } \\
>=1.5^{*} \text { Crec. ventas } \\
\text { país }\end{array}$ \\
\hline Estrella & Mayor al 25\% & $\begin{array}{l}\text { Crec. ventas sector } \\
>=1.5^{*} \text { Crec. ventas } \\
\text { país }\end{array}$ \\
\hline Vaca & Mayor al 25\% & $\begin{array}{l}\text { Crec. ventas sector } \\
<1.5^{*} \text { Crec. ventas país }\end{array}$ \\
\hline
\end{tabular}

Fuente: Elaboración propia.

- Crecimiento del sector: para medir el crecimiento del sector, se comparó el crecimiento de las ventas 
de la industria a la que pertenece la empresa con el crecimiento de las ventas totales de todos los sectores. Se definió un sector con alto crecimiento a aquel sector con una tasa de crecimiento superior a la tasa de crecimiento total en un $50 \% 2$.

\subsection{Descripción de las variables}

Transición: la variable dependiente se ha construido de tal forma que permita medir los cambios en la posición de mercado entre tipos de empresa. Las categorías de esta variable son las siguientes:

- Empresas (de cualquier tipo) que no se mueven se clasifican como 0.

- Empresas tipo Vaca que desmejoran se clasifican como 1.

- Empresas tipo Estrella que desmejoran se clasifican como 2.

- Empresas tipo Interrogante que desmejoran se clasifican como 3.

- Empresas tipo Perro que mejoran se clasifican como 4.

- Empresas tipo Interrogante que mejoran se clasifican como 5.

- Empresas tipo Estrella que mejoran se clasifican como 6.

EGO (Efectivo Generado por la Operación): es el flujo de caja que queda disponible después de atender los compromisos tributarios y de reposición de capital de trabajo. Es, por tanto, el efectivo que queda disponible para atender tres compromisos: inversión en activos fijos, servicio a la deuda y reparto de dividendos. Si se utiliza el ratio EGO/Ventas, el cual es un indicador que representa los centavos que quedan disponibles por cada peso de ventas para atender los tres compromisos señalados arriba, se tiene una medida de eficiencia y de liquidez. Lo ideal es tener este cociente lo más alto posible. En la regresión se utiliza la relación de EGOV de la empresa-EGOV Total del Sector.

\footnotetext{
2 Uno de los requisitos para ser sector líder en una economía es que el crecimiento del sector sea superior al de la economía. El valor de crecimiento del sector respecto a la economía fijado para este trabajo es responsabilidad de los autores.
}

EBITDA: es definido como la utilidad operativa más cualquier partida que no implique desembolso real de efectivo; generalmente, estas partidas son las depreciaciones y las amortizaciones; es por tanto la verdadera utilidad que finalmente se convierte en efectivo para la empresa. En la regresión se utiliza la relación de EBITDA de la empresa-EBITDA Total del Sector.

KTNO (Capital de Trabajo Neto): es definido como la suma de las cuentas por cobrar, más los inventarios, menos las cuentas por pagar a proveedores de bienes y servicios. Este es el capital que la empresa requiere para llevar a cabo las operaciones día a día. El cociente entre el KTNO y las ventas es conocido como la productividad del capital de trabajo (PKT) y refleja los centavos que por cada peso de ventas la empresa debe mantener invertidos en capital de trabajo, por tanto, es una medida clara del grado de liquidez de la empresa. Lo ideal es tener una PKT lo más baja posible. En la regresión se utiliza la relación de PKT de la empresa - PKT Total del Sector.

AF (Activos Fijos): también conocidos como la propiedad, planta y equipo. Los AF son los activos de largo plazo que la empresa requiere para operar. Este tipo de activos varía de acuerdo con las características del sector y del negocio de la empresa. Se utiliza el cociente entre los activos fijos y el activo total. En la regresión se utiliza la relación de Activos Fijos de la empresa-Activos Fijos Total del Sector.

Tamaño de la empresa: se ha asignado un valor categórico a cada empresa según su tamaño; este procedimiento se ha hecho con base en los parámetros fijados por la SSC para definir el tamaño de una empresa. Según este organismo, las empresas pueden ser clasificadas por tamaño de la siguiente forma:

- Empresas micro y pequeñas: activos menores a 5.000 millones.

- Empresas medianas: activos entre 5.001 y 30.000 millones.

- Empresas grandes: activos superiores a 30.000 millones. 
Edad: la edad de la empresa se construye como la diferencia de tiempo entre la fecha de registro de la empresa y la fecha en el período de estudio.

Costos hundidos: esta variable hace referencia a la presencia de altos costos de entrada en un sector y se calcula como el promedio por sector del cociente entre la propiedad, planta y equipo sobre los activos totales. Un alto valor de esta variable implica que existen altos costos de entrada en activos fijos dentro de un sector, y pueden representar costos hundidos para las firmas que deseen entrar al sector. En la medida en que existan altos costos de este tipo en un sector las barreras de entrada y salida serán mayores, por lo que la concentración del mercado será mayor y la intensidad de la competencia podrá ser más baja.

Altas inversiones: esta variable se calcula como el promedio por sector del cociente entre el KTNO y los activos totales. La intuición detrás de esta variable se relaciona con el grado de inversión en capital de trabajo requerida en un sector; valores altos podrán funcionar como una barrera de entrada al demandar una alta inversión inicial para iniciar las operaciones.

HHI (Índice de Herfindahl-Hirschman): es considerado en la literatura de la organización industrial como una medida de concentración de una industria y de la rentabilidad de un sector. Se calcula como la sumatoria de las participaciones de mercado al cuadrado; según Church y Ware (2000), si el índice se acerca a cero se dice que el mercado tiene una estructura competitiva, mientras que si se acerca a uno se dice que tiene una estructura monopolística. Este índice ha sido calculado teniendo en cuenta el mercado total nacional según código CIIU a cuatro dígitos como el mercado relevante de una empresa. Al incluir el índice de Herfindahl-Hirschman se pretende capturar parte de la dinámica microeconómica del sector, especialmente la estructura del mercado y el grado de competencia.

Escala: esta variable es igual al número total de empresas en un mismo sector en un determinado departamento. Esto se hace con el fin de determinar si la aglomeración de empresas produce externalidades positivas o negativas en la economía. En caso de ser positivas el efecto se da a través de spillovers positivos de conocimiento. Brandenburger y Nalebuff (1996) sostienen que las interacciones entre firmas pueden tener efectos positivos, aparte de los efectos negativos que trae una mayor intensidad de la competencia. Por otro lado, si las externalidades son negativas, el efecto será a través del efecto de intensidad de la competencia.

\subsection{Estadísticas descriptivas}

A continuación se presentan las tablas con estadísticas descriptivas y el análisis de las mismas. Para las variables que se encuentra en cantidades absolutas los valores están en miles de pesos colombianos. La construcción de las diferentes variables permite hacer análisis tanto de las empresas como tal, como de su posición de mercado relativa en el interior del sector al que pertenecen. En primera instancia, se presentan los resultados de las variables de las empresas, para luego mostrarlas en relación con el sector donde la firma se encuentra.

En la tabla 2 se puede inferir que no hay grandes diferencias por edad entre los tipos de empresas, todas tienen una alta necesidad de liquidez, reflejada en el indicador de palanca de crecimiento (PDC), lo que es coherente con el indicador "altas inversiones" que se observa en la tabla 4, que dice que todas estas empresas exigen una gran liquidez en el corto plazo para cumplir las obligaciones y alcanzar la máxima productividad posible.

En relación con el nivel de activos, es notable cómo el activo fijo en ningún caso supera el 20\% de los activos totales de las empresas, como se observa en la Tabla 3, al igual que los costos hundidos generados por estos como inversión inicial, sin presentarse mucha diferencia tanto entre los tipos de empresa como dentro de cada tipo (ver tabla 4); no obstante, como se observa en la tabla 3, la generación de efectivo a través de la opera- 
Tabla 2. Estadística descriptiva de las empresas: variables generales

\begin{tabular}{|c|c|c|c|c|c|}
\hline Tipo & Estadísticos & Activo & Ventas & Tamaño & Edad \\
\hline \multirow{2}{*}{$\begin{array}{l}\text { Perro } \\
\text { (1618 obs.) }\end{array}$} & Promedio & $14,900,000$ & $16,000,000$ & 1.7664 & 27 \\
\hline & Desviación Estándar & $29,800,000$ & $28,000,000$ & 0.5862 & 13 \\
\hline \multirow{2}{*}{$\begin{array}{l}\text { Interrogante } \\
\text { (506 obs.) }\end{array}$} & Promedio & $23,100,000$ & $20,500,000$ & 1.8478 & 26 \\
\hline & Desviación Estándar & $72,300,000$ & $31,000,000$ & 0.6154 & 12 \\
\hline \multirow{2}{*}{$\begin{array}{l}\text { Estrella } \\
\text { (338 obs.) }\end{array}$} & Promedio & $94,200,000$ & $94,500,000$ & 2.2840 & 28 \\
\hline & Desviación Estándar & $294,000,000$ & $248,000,000$ & 0.6776 & 14 \\
\hline \multirow{2}{*}{$\begin{array}{l}\text { Vaca } \\
\text { (986 obs.) }\end{array}$} & Promedio & $72,900,000$ & $77,000,000$ & 2.3661 & 31 \\
\hline & Desviación Estándar & $132,000,000$ & $191,000,000$ & 0.6598 & 15 \\
\hline
\end{tabular}

Fuente: Elaboración propia.

Tabla 3. Estadística descriptiva de las empresas: variables financieras

\begin{tabular}{|l|l|c|c|c|c|}
\hline \multicolumn{1}{|c|}{ Tipo } & \multicolumn{1}{c|}{ Estadísticos } & EGO & EBITDA & PKT & $\begin{array}{c}\text { Activo Fijo / } \\
\text { Activo Total }\end{array}$ \\
\hline \multirow{2}{*}{$\begin{array}{l}\text { Perro } \\
(1618 \text { obs.) }\end{array}$} & Promedio & $1,126,137$ & $1,389,555$ & 0.7854 & 0.1826 \\
\cline { 2 - 6 } & Desviación Estándar & $2,518,866$ & $3,296,566$ & 0.7150 & 0.1509 \\
\hline $\begin{array}{l}\text { Interrogante } \\
\text { (506 obs.) }\end{array}$ & Promedio & $1,634,682$ & $1,702,981$ & 0.9439 & 0.1592 \\
\cline { 2 - 6 } & Desviación Estándar & $3,787,107$ & $3,707,065$ & 1.3525 & 0.1418 \\
\hline $\begin{array}{l}\text { Estrella } \\
\text { (338 obs.) }\end{array}$ & Promedio & $15,500,000$ & $15,700,000$ & 0.7515 & 0.1037 \\
\cline { 2 - 6 } $\begin{array}{l}\text { Vaca } \\
(986 \text { obs.) }\end{array}$ & Desviación Estándar & $67,900,000$ & $69,500,000$ & 0.7224 & 0.1285 \\
\cline { 2 - 6 } & Promedio & $8,443,446$ & $9,803,097$ & 0.7140 & 0.1924 \\
\hline
\end{tabular}

Fuente: Elaboración propia.

Tabla 4. Estadística descriptiva de las empresas: variables de estructura de mercado

\begin{tabular}{|c|c|c|c|c|c|}
\hline Tipo & Estadísticos & Costos Hundidos & Altas Inversiones & $\mathrm{HHI}$ & Escala \\
\hline \multirow{2}{*}{$\begin{array}{l}\text { Perro } \\
\text { (1618 obs.) }\end{array}$} & Promedio & 0.1803 & 0.7471 & 0.1585 & 12 \\
\hline & Desviación Estándar & 0.0843 & 0.2556 & 0.1452 & 13 \\
\hline \multirow{2}{*}{$\begin{array}{l}\text { Interrogante } \\
\text { (506 obs.) }\end{array}$} & Promedio & 0.1588 & 0.7501 & 0.1831 & 11 \\
\hline & Desviación Estándar & 0.0867 & 0.2355 & 0.1722 & 10 \\
\hline \multirow{2}{*}{$\begin{array}{l}\text { Estrella } \\
\text { (338 obs.) }\end{array}$} & Promedio & 0.1837 & 0.7321 & 0.3925 & 6 \\
\hline & Desviación Estándar & 0.1103 & 0.2449 & 0.2897 & 7 \\
\hline \multirow{2}{*}{$\begin{array}{l}\text { Vaca } \\
\text { (986 obs.) }\end{array}$} & Promedio & 0.1970 & 0.6789 & 0.2887 & 7 \\
\hline & Desviación Estándar & 0.0952 & 0.2555 & 0.2711 & 8 \\
\hline
\end{tabular}

Fuente: Elaboración propia 
ción normal de la empresa y las ganancias antes de obligaciones son mayores en las empresas con mayor participación de mercado, en especial las tipo Estrella, lo que concuerda con su descripción respecto a ser empresas que generan altos ingresos aunque a un costo relativamente alto.

Desde los indicadores que caracterizan los sectores, se encuentra que las empresas con mayor participación de mercado (Estrellas y Vacas) tienen un índice de HHI más alto, es decir, tienen más poder de mercado (ver tabla 4); por demás, este tipo de empresas son mayoritariamente medianas y grandes, a diferencia de las empresas Perro, donde las empresas pequeñas tienen una mayor importancia, junto con las medianas.

Respecto a las variables que muestran la relación de la empresa con su sector, se observa en la tabla 5 que:

- Tanto las empresas Perro como las Interrogantes son pequeñas en comparación con su sector; las empresas más grandes, en este sentido, se ubican entre las empresas Vaca.

- Es notable, entre los resultados descriptivos, cómo las empresas Interrogantes tienen la menor producción relativa en relación con su sector, debido a su situación de poca participación en la industria.
- El capital de trabajo neto operativo relativo muestra que las empresas con mayor liquidez en comparación con su sector son las empresas tipo Estrella y Vaca, lo que es muy importante en las primeras debido a su mayor necesidad de financiación en el corto plazo.

- Las empresas Perro e Interrogante, a diferencia de las tipo Estrella y Vaca, tienen más deuda que reservas.

\section{METODOLOGÍA}

Para la realización de este ejercicio, se procedió en el siguiente orden: primero, se construyeron algunos indicadores financieros con base en la información obtenida, los cuales pudieron ser observados en la sección anterior; luego, se organizaron los datos por categorías según lo establecido en la matriz de crecimiento-participación. Así, se obtuvieron cuatro categorías: empresas "Perro", "Interrogante", "Estrella" y "Vaca"; tercero, se creó una variable que permite identificar a través del tiempo algún cambio en la posición de mercado de las empresas dada esta clasificación; finalmente se aplicó un modelo de selección multinomial sobre la muestra, que permite identificar las variables más importantes para explicar el cambio en cuestión.

Tabla 5. Estadística descriptiva de las empresas relativas al sector que pertenecen: variables financieras

\begin{tabular}{|l|l|c|c|c|c|}
\hline \multicolumn{1}{|c|}{ Tipo } & \multicolumn{1}{|c|}{ Estadísticos } & EBITDA & PKT & PDC & EGOV \\
\hline \multirow{2}{*}{$\begin{array}{l}\text { Perro } \\
(1618 \text { obs.) }\end{array}$} & Promedio & 0.0234 & 1.2221 & 1.4463 & 0.0219 \\
\cline { 2 - 5 } & Desviación Estándar & 0.0895 & 0.8126 & 8.8114 & 0.1687 \\
\hline $\begin{array}{l}\text { Interrogante } \\
(506 \text { obs.) }\end{array}$ & Promedio & 0.0278 & 1.4180 & 1.6478 & 0.0580 \\
\cline { 2 - 5 } $\begin{array}{l}\text { Estrella } \\
(338 \text { obs.) }\end{array}$ & Desviación Estándar & 0.0867 & 1.6898 & 7.0712 & 1.5818 \\
\hline Vaca & Promedio & 0.3533 & 1.0452 & 0.9788 & 0.3608 \\
\cline { 2 - 5 }$(986$ obs.) & Desviación Estándar & 0.4480 & 0.4237 & 2.8897 & 2.2716 \\
\cline { 2 - 6 } & Promedio & 0.2802 & 1.0406 & 1.3534 & 0.2446 \\
\hline
\end{tabular}

Fuente: Elaboración propia. 
La información ha sido organizada de forma tal que pueda ser tratada como un corte transversal. Las empresas que la componen se caracterizan por tener diferencias entre ellas, debido a que pertenecen a distintos sectores, lo que crea diversas combinaciones de capital fijo y variable, activos, proveedores, etc. Esto exige su ordenación para una mejor identificación de los efectos de pertenecer a uno u otro sector, y a algún departamento específico. Dado lo anterior, el modelo para el caso de la variable dependiente "Transición" es de la forma:

$$
Y_{i}=\left\{\begin{array}{lllll}
0, \text { No hay } & \text { cambios } & & & \\
\text { 1, Cambio } & \text { Negativo } & \text { de } & \text { empresa } & \text { Vaca } \\
\text { 2, Cambio } & \text { Negativo } & \text { de } & \text { empresa } & \text { Estrella } \\
\text { 3, Cambio } & \text { Negativo } & \text { de } & \text { empresa } & \text { Interrogante } \\
\text { 4, Cambio } & \text { Positivo } & \text { de } & \text { empresa } & \text { Perro } \\
\text { 5, Cambio } & \text { Positivo } & \text { de } & \text { empresa } & \text { Interrogante } \\
\text { 6, Cambio } & \text { Positivo } & \text { de } & \text { empresa } & \text { Estrella }
\end{array}\right\}
$$

Para estimar la probabilidad de estar en alguna de estas categorías, se utiliza un modelo logit multinomial. El modelo se puede expresar como:

$$
\begin{aligned}
& P_{i j}=P\left(Y_{i}=j \mid X_{i}\right)=F_{j}\left(X_{i}^{\prime} \beta\right), \\
& j=0, \ldots, 6, i=1,2, \ldots, 3.448
\end{aligned}
$$

Donde la especificación será:

$$
P_{i j}=\frac{e^{x_{i} \beta_{j}}}{\sum_{l=1}^{M} e^{x_{i} \beta_{l}}}
$$

Donde "i" son las empresas, "j" y "l" las diferentes categorías. Sin embargo, la interpretación de los estimadores conseguidos en este procedimiento no puede hacerse de forma directa, por lo que hay que conseguir información complementaria, como la comparación de los datos obtenidos desde una categoría base ${ }^{3}$ y los efectos marginales ${ }^{4}$.

3 Esto se hace a través de proporciones de riesgo. Su especificación es $\frac{P\left(Y_{i}=j\right)}{P\left(Y_{i}=\text { categoríabase }\right)}=e^{x_{i} \beta_{j}}$, lo que significa el cambio proporcional en el riesgo de escoger la alternativa "j" en vez de la categoría base cuando hay un cambio en una unidad en xi(ver Cameron y Trivedi, 2009, p. 486).

$4 \quad$ Se especifican como $\frac{\partial P_{i j}}{\partial x_{i}}=P_{i j}\left(\beta_{j}-\overline{\beta_{j}}\right)$, significando el efecto de un cambio unitario en el regresor sobre la j-ésima probabilidad (ver Cameron y Trivedi, 2009, p. 488).
Este tipo de modelos se estiman con el método de máxima verosimilitud (MV), que contribuye a crear estimaciones consistentes de los parámetros de la regresión, como lo señalan Cameron y Trivedi (2005, Cap. 15). Para el caso de los modelos multinomiales, se supone independencia entre las empresas, la función de verosimilitud está dada por la siguiente expresión:

$$
L_{n}=\prod_{i=1}^{N} \prod_{j=0}^{M} P_{i j}^{Y_{i j}}
$$

Donde " $\mathrm{N}$ " es el número de empresas analizadas y "M" el número de categorías.

Los estimadores obtenidos se distribuirán asintóticamente de la siguiente forma:

$$
\hat{\beta} \sim N\left[\beta,\left(\sum_{i=1}^{N} \sum_{j=1}^{M} \frac{1}{P_{i j}} \frac{\partial P_{i j}}{\partial \beta} \frac{\partial P_{i j}}{\partial \beta^{\prime}}-\frac{\partial^{2} P_{i j}}{\partial \beta \partial \beta^{\prime}}\right)^{-1}\right]
$$

\section{MODELO Y RESULTADOS}

En esta sección se explica el modelo econométrico utilizado, el cual tiene como objetivo analizar las transiciones desagregadas; se discrimina por tipo de empresa y se tiene en cuenta los cambios ascendentes o descendentes según tipo. La variable dependiente de este modelo es "Transición", y la categoría base de comparación es no experimentar transición.

Al modelo planteado se le realizaron pruebas de significancia individual, multicolinealidad e independencia de alternativas irrelevantes. A través de las denominadas pruebas de Wald (ver Anexo A) sobre la significancia conjunta de los regresores en las diversas categorías se observa que las variables Productividad de Capital de Trabajo (PKT) y escala no son significativas conjuntamente para las diversas categorías; el resto de los regresores son significativos, aunque se aprecia que las variables asociadas a la estructura del mercado son más significativas que las variables financieras. En la Tabla 6 se enseña la probabilidad de cambio de estado en la posición de mercado estimada por el 
modelo y la frecuencia relativa observada de este acontecimiento. A modo de ejemplo, la frecuencia relativa observada de ascenso de las empresas tipo Perro es 19\%, en tanto que la probabilidad estimada de dicho cambio es 20\%, la máxima probabilidad estimada entre todas las empresas tipo Perro de evidenciar el cambio de estado ascendente es $91 \%$.

Se aprecia que el modelo se ajusta aceptablemente a los datos, aunque la predicción de la categoría que hace referencia al cambio descendente en la posición de mercado de las empresas tipo Vaca no es muy buena; podría decirse que el modelo predice mejor los cambios ascendentes que los descendentes. El modelo es particularmente potente para predecir los cambios positivos de empresas tipo Perro y tipo Estrella. Adicionalmente se ha hecho la prueba de multicolinealidad (ver Anexo B) y se puede concluir que no existen problemas de este tipo entre los regresores, y finalmente, la prueba de independencia de alternativas irrelevantes realizada a través del test de Hausman (1978) indica el cumplimiento de dicho supuesto (ver Anexo C).

En las tablas 7 y 8 se muestran los resultados del modelo logit multinomial para los diferentes casos de cambio en la posición de mercado. Específicamente, las empresas tipo Perro solo tienen la posibilidad de ascender, pues no existe un nivel inferior al que puedan bajar. En este caso los regresores asociados a barreras de entrada tienen un fuerte efecto negativo sobre la probabilidad de ascender. La relación Activo Fijo firma-Activo Fijo Sector incrementa levemente la probabilidad de que una empresa tipo Perro experimente un cambio en la posición de mercado positiva. Tanto la edad como el tamaño también reducen la probabilidad de cambio hacia arriba, lo que indica que las empresas maduras y más grandes de este tipo tienden a permanecer estables. Por otra parte, el resultado asociado al HHI implica que una mayor concentración del mercado reduce la probabilidad de avanzar positivamente.

Tabla 6. Probabilidades estimadas y frecuencia relativa observada

\begin{tabular}{|l|c|c|c|c|}
\hline \multicolumn{1}{|c|}{ Variable } & Mean & Dev. St. & Min & Max \\
\hline \multicolumn{5}{|c|}{ Probabilidades estimadas por el modelo } \\
\hline 0: Transición Nula & 0.53 & 0.13 & 0.00 & 1.00 \\
\hline 1: Descenso de Vaca & 0.12 & 0.08 & 0.00 & 0.53 \\
\hline 2: Descenso de Estrella & 0.03 & 0.04 & 0.00 & 0.82 \\
\hline 3: Descenso de Interrogante & 0.07 & 0.07 & 0.00 & 1.00 \\
\hline 4: Ascenso de Perro & 0.20 & 0.14 & 0.00 & 0.91 \\
\hline 5: Ascenso de Interrogante & 0.02 & 0.06 & 0.00 & 1.00 \\
\hline 6: Ascenso de Estrella & 0.04 & 0.06 & 0.00 & 0.92 \\
\hline \multicolumn{7}{|c|}{ Frecuencias observadas } & & \\
\hline 0: Transición Nula & 0.54 & 0.50 & 0.00 & 1.00 \\
\hline 1: Descenso de Vaca & 0.02 & 0.15 & 0.00 & 1.00 \\
\hline 2: Descenso de Estrella & 0.06 & 0.23 & 0.00 & 1.00 \\
\hline 3: Descenso de Interrogante & 0.14 & 0.34 & 0.00 & 1.00 \\
\hline 4: Ascenso de Perro & 0.19 & 0.39 & 0.00 & 1.00 \\
\hline 5: Ascenso de Interrogante & 0.02 & 0.14 & 0.00 & 1.00 \\
\hline 6: Ascenso de Estrella & 0.03 & 0.18 & 0.00 & 1.00 \\
\hline
\end{tabular}

Fuente: Elaboración propia

${ }^{* * *}$ Significativo al $1 \% * *$ Significativo al 5\%

*Significativo al 10\% 
Tabla 7. Resultados modelo logit multinomial

\begin{tabular}{|c|c|c|c|c|c|c|}
\hline Transición & Coef. & Std. Err. & Sig. & Coef. & Std. Err. & Sig. \\
\hline \multicolumn{4}{|c|}{ Descenso de Vaca } & \multicolumn{3}{|c|}{ Ascenso de Perro } \\
\hline EGOV & 0.0007 & 0.0019 & & -0.0013 & 0.0028 & \\
\hline EBITDA & -0.0050 & 0.0038 & & -0.0107 & 0.0039 & $* * *$ \\
\hline PKT & 0.0011 & 0.0009 & & 0.0011 & 0.0007 & \\
\hline Afijo & 0.0161 & 0.0054 & $* * *$ & W & 0.0062 & \\
\hline Edad & -0.0050 & 0.0057 & & -0.0327 & 0.0061 & $* * *$ \\
\hline Tamaño 2 & 0.2086 & 0.2903 & & -0.2247 & 0.1924 & \\
\hline Tamaño 3 & 0.8930 & 0.3018 & $* * *$ & -1.0182 & 0.2474 & $* * *$ \\
\hline Costos Hundidos & -0.0354 & 0.0119 & $* * *$ & -0.0962 & 0.0122 & $* * *$ \\
\hline Altas Inversiones & -0.0066 & 0.0040 & * & -0.0170 & 0.0037 & $* * *$ \\
\hline $\mathrm{HHI}$ & 0.4981 & 0.4072 & & -1.0929 & 0.3953 & $* * *$ \\
\hline Escala & 0.0052 & 0.0097 & & -0.0019 & 0.0086 & \\
\hline \multicolumn{4}{|c|}{ Descenso de Interrogante } & \multicolumn{3}{|c|}{ Ascenso de Interrogante } \\
\hline EGOV & -0.0090 & 0.0041 & $* *$ & -0.0133 & 0.0045 & $* * *$ \\
\hline EBITDA & -0.0074 & 0.0058 & & -0.0011 & 0.0095 & \\
\hline PKT & -0.0001 & 0.0015 & & -0.0043 & 0.0058 & \\
\hline Afijo & -0.0386 & 0.0146 & $* * *$ & -0.0035 & 0.0239 & \\
\hline Edad & -0.0278 & 0.0101 & $* * *$ & -0.1181 & 0.0293 & $* * *$ \\
\hline Tamaño 2 & -0.1589 & 0.3075 & & 0.9376 & 1.0975 & \\
\hline Tamaño 3 & -1.3741 & 0.4515 & $* * *$ & 2.0041 & 1.0874 & * \\
\hline Costos Hundidos & 0.0654 & 0.0241 & $* * *$ & 0.0987 & 0.0495 & $* *$ \\
\hline Altas Inversiones & 0.0169 & 0.0087 & $*$ & 0.0331 & 0.0191 & $*$ \\
\hline $\mathrm{HHI}$ & 1.0529 & 0.601 & * & -0.9296 & 1.4234 & \\
\hline Escala & -0.0033 & 0.0142 & & -0.0069 & 0.0336 & \\
\hline \multicolumn{4}{|c|}{ Descenso de Estrella } & \multicolumn{3}{|c|}{ Ascenso de Estrella } \\
\hline EGOV & -0.0061 & 0.0036 & $*$ & 0.0008 & 0.0021 & \\
\hline EBITDA & -0.0132 & 0.0052 & $* *$ & 0.0078 & 0.0047 & * \\
\hline PKT & 0.0023 & 0.0009 & $* *$ & -0.0027 & 0.0033 & \\
\hline Afijo & 0.0046 & 0.0131 & & 0.0128 & 0.0077 & $*$ \\
\hline Edad & -0.0200 & 0.0129 & & -0.0081 & 0.0099 & \\
\hline Tamaño 2 & -0.9326 & 0.4065 & $* *$ & -0.0046 & 0.5709 & \\
\hline Tamaño 3 & -0.4290 & 0.4469 & & 1.0646 & 0.5725 & * \\
\hline Costos Hundidos & -0.0120 & 0.0258 & & 0.0166 & 0.0188 & \\
\hline Altas Inversiones & -0.0078 & 0.0088 & & 0.0014 & 0.0076 & \\
\hline $\mathrm{HHI}$ & -1.0799 & 0.8562 & & -0.334 & 0.7950 & \\
\hline Escala & -0.0948 & 0.0375 & $* *$ & -0.0643 & 0.0323 & $* *$ \\
\hline
\end{tabular}

Fuente: Elaboración propia

***Significativo al 1\%**Significativo al 5\%

*Significativo al 10\% 
En el caso de las empresas Interrogante se pueden experimentar ascensos y descensos. Para el caso de las transiciones positivas, sale a la vista un resultado curioso asociado a los regresores de barreras de entrada. En este caso, la probabilidad de que una empresa tipo Interrogante experimente una transición ascendente está asociada positivamente con el grado de barreras de entrada, lo que refleja que este tipo de imperfecciones favorecen probablemente a las empresas que están en la cima en relación con las empresas rezagadas. Por otro lado, la edad se asocia negativamente con la probabilidad de ascender. Según esto, a medida que una empresa envejece los rendimientos marginales son cada vez más decrecientes, por lo que la empresa es menos eficiente y tiene menos probabilidad de tener un cambio positivo.

Para el caso de las transiciones negativas, la relación Activo Fijo firma-Activo Fijo Sector reduce la probabilidad de que una empresa tipo Interrogante caiga. Así mismo, la relación EGO Ventas-EGO Ventas Sector tiene un efecto similar. Las empresas que tienen una mayor capacidad de generar un alto flujo de efectivo como proporción de las ventas y de adelantar procesos de expansión adquieren ventajas relativas frente a los competidores y, por tanto, tienen menor probabilidad de descender. Nuevamente, los costos hundidos y las altas inversiones tienen un fuerte efecto sobre la probabilidad, en este caso, de descender. Adicionalmente, el efecto de la competencia es decisivo sobre la probabilidad; el resultado de las barreras de entrada y del HHI sugiere que los mercados concentrados generan grandes asimetrías entre grandes y pequeños, donde estos últimos son los más afectados. Aquí la edad tiene un efecto modesto aunque negativo, mientras que el tamaño sí presenta un fuerte efecto negativo sobre la probabilidad. Por último, las empresas grandes tipo Interrogante tienen aparentemente una fuerte ventaja sobre las empresas pequeñas del mismo tipo, pues estas últimas tienen una mayor probabilidad de descender.
Para las empresas tipo Estrella se puede ascender y descender. Para el caso de las transiciones positivas, solo la relación EBITDA firma-EBITDA Sector, los costos hundidos y escala tienen efectos significativos sobre la probabilidad. Un incremento en la relación EBITDA firma-EBITDA Sector aumenta la probabilidad de que una empresa tipo Estrella ascienda; las firmas que generan un alto volumen de efectivo tienen una mayor probabilidad de convertirse en empresas tipo Vaca. El efecto de los Costos Hundidos es similar al evidenciado en el caso de las empresas tipo Interrogante. La variable escala reduce en este caso la probabilidad de ascender, lo que sugiere que los efectos de la aglomeración de empresas en una región son de hecho de tipo competencia: por cada empresa nueva (por sector y región), las empresas tipo Estrella tienen una menor probabilidad de ascender.

Para el caso de las transiciones negativas, la relación EBITDA firma-EBITDA Sector reduce la probabilidad de que una empresa tipo Estrella descienda. Igualmente, la variable escala reduce la probabilidad de descender. Aunque pareciera contradictorio con el resultado obtenido para esta misma variable en el caso de las transiciones positivas, el resultado es bastante interesante pues sugiere que a medida que el número de empresas aumenta el mercado se vuelve menos dinámico y más estable.

Finalmente, para las empresas tipo Vaca, al estar ubicadas en la parte más alta de la escala, estas empresas sólo pueden presentar transiciones descendentes. La relación Activo Fijo firma-Activo Fijo Sector aumenta la probabilidad de que una empresa tipo Vaca descienda. Lejos de ser contra intuitivo, este resultado indica que una vez las empresas alcanzan su tope natural de crecimiento, las inversiones de expansión no son necesarias, pues el mercado mismo ya tiene pocas posibilidades de crecer. De hecho, las empresas tipo Vaca que mantienen altos volúmenes en activos fijos experimentan una mayor probabilidad de descender pues estos activos podrían ser invertidos en actividades 
Tabla 8. Promedio de los efectos marginales

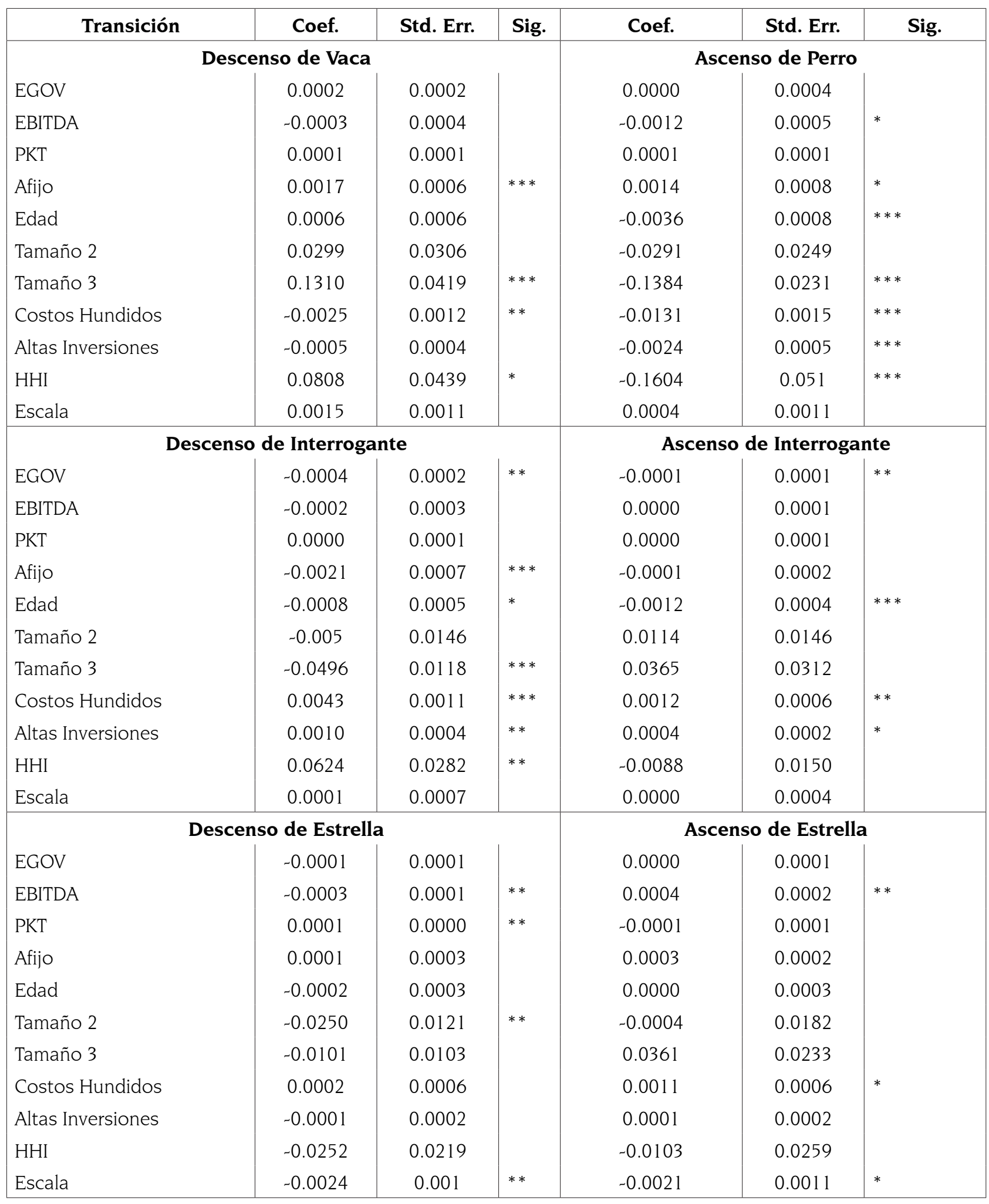

Fuente: Elaboración propia. 
más rentables. Las empresas grandes tipo Vaca tienen aparentemente una fuerte desventaja frente a las empresas pequeñas de este tipo. Una posible explicación de esto es que las empresas tipo Vaca, al no tener más oportunidades de crecimiento, no se benefician de las ventajas absolutas que representa tener un mayor tamaño; por el contrario, en esta etapa las empresas más pequeñas pueden tener ventajas asociadas a una mayor eficiencia y menores niveles de capacidad ociosa.

\section{CONCLUSIONES}

Los resultados que se presentaron en la sección anterior permiten deducir dos conclusiones interesantes. Primero, las variables financieras generalmente tienen efectos similares sobre las empresas: aunque de diferentes magnitudes, puede decirse que la mayoría de las empresas se benefician de una alta generación de flujo de caja (EBITDA) y de ser eficientes en la generación de efectivo (EGOV). Además, la inversión en activos fijos generalmente reduce la probabilidad de descender o aumenta la de ascender -según el caso- a excepción de las empresas tipo Vaca, donde esta variable de hecho aumenta la probabilidad de que estas empresas tengan una transición negativa: las empresas tipo Vaca que mantienen altos volúmenes de activos fijos, experimentan una mayor probabilidad de descender pues estas inversiones en este punto comienzan a demandar flujos de caja mayores que podrían ser invertidos en actividades más rentables. Dado que la relación PKT Firma-PKT Sector no resultó significativa, se puede concluir que es más importante el volumen generado de caja por la empresa en relación con el sector (EBITDA y EGOV) que la cantidad de caja demandada para operar en relación con el sector (PKT). Este resultado tiene sentido, pues generalmente la cantidad de caja requerida por el negocio está determinada más por factores generales del sector que por políticas financieras específicas de las empresas. Esto revela que las empresas más grandes, que logran atender una mayor porción del mercado tienen una importante ventaja sobre el resto. Respecto a la edad de las empresas, los resultados sugieren que las empresas más viejas tienden a ser más estables, mientras que las empresas más jóvenes experimentan una mayor transición, tanto hacia arriba como hacia abajo.

El segundo resultado interesante tiene que ver con las variables relacionadas con la estructura del mercado. Los resultados de las variables asociadas a las barreras de entrada llaman la atención porque tienen efectos completamente diferentes, de acuerdo con del tipo de empresa. Por ejemplo, mientras que un mayor grado de barreras de entrada reduce la probabilidad de que una empresa tipo Vaca tenga una transición negativa, para el caso de las empresas tipo Interrogante, el aumento en el nivel de barreras de entrada tiene un fuerte efecto positivo sobre la probabilidad de descender. Particularmente, en el caso de las empresas tipo Interrogante, las barreras de entrada generan dinamismo en el mercado, ya que las empresas más sólidas se favorecen y ascienden, mientras que las empresas más débiles descienden.

Un aumento en el tamaño de una empresa tiene efectos diferentes de acuerdo con el tipo de empresa. Para el caso de las empresas tipo Vaca, al no poder crecer más, un aumento de su tamaño incrementa la probabilidad de descender, posiblemente debido a que se generan ineficiencias o capacidades ociosas. Para el resto de empresas, un incremento en el tamaño reduce la probabilidad de que se experimente una transición negativa, pero también reduce la probabilidad de una transición positiva. Esto sugiere que las empresas que cambian de estado son generalmente las empresas pequeñas, mientras que las medianas y grandes tienden a mantenerse en su estado original, lo que parece decir que las empresas Perro - donde está la mayoría de las empresas pequeñas- comúnmente salen de este estado. 
Los resultados de las variables asociadas a la estructura de mercado llaman la atención. El regresor HHI resulta significativo en ambas categorías (ascenso y descenso). Un incremento de este índice aumenta la probabilidad de que una empresa experimente una transición negativa y reduce la probabilidad de una positiva. A medida que la concentración del mercado aumenta, es más difícil para una empresa avanzar, pues la brecha entre ella y el líder del mercado se incrementa, y este gana cada vez más poder para implementar estrategias que impidan que otros competidores lo alcancen. En general se desprende la conclusión de que los mercados más concentrados son también los mercados en donde se esperaría observar menores eventos de transición; son sectores de lenta evolución.

Finalmente, dadas las conclusiones presentadas arriba sobre los resultados del modelo, se pueden hacer las siguientes recomendaciones de política. Específicamente, se ha optado por concentrar esta última discusión en las empresas Perro y las empresas Interrogante; es decir, en el lado derecho de la figura 1. Además, dado que las recomendaciones están orientadas al hacedor de política económica o regulador, solo se hacen los respectivos análisis respecto a las variables que este puede efectivamente manipular. Por esto, se decide únicamente hacer ejercicios de política con base en variables asociadas a la edad, el tamaño y la concentración de mercado.

En el caso específico de las empresas Perro, que solo les queda ascender, se pudo observar cómo aquellas que son pequeñas, jóvenes, y que se encuentran en mercados altamente competitivos son las que tienen mayor probabilidad de tener un cambio positivo en su posición de mercado. Esto se puede observar en los anexos D, E y F. Así, una empresa hipotética con un año de edad, de tamaño pequeño y que opera en un mercado perfectamente competitivo $(\mathrm{HHI}=0)$ tendría una probabilidad de ascenso del 14.3\%; para una empresa hipotética promedio, es decir, pequeña y con el resto de re- gresores en los valores medios, la probabilidad de ascender es únicamente de 8.3\%. Dado lo anterior, una política efectiva de promoción a empresas de bajo crecimiento y baja participación debe estar orientada a empresas pequeñas, jóvenes y que operan en mercados altamente competitivos.

Respecto a las empresas Interrogante, se debe tener en cuenta que estas pueden tanto ascender a una situación de empresas Estrella o descender a empresas Perro. En el primer caso, la probabilidad de ascenso es mayor para empresas jóvenes, grandes y en mercados competitivos como se puede observar en los anexos G, H, y I; en el segundo caso, la probabilidad de descender es menor para empresas viejas, grandes y que se encuentran en mercados competitivos, como se refleja en los anexos J, K y L. De esta manera, si una empresa hipotética con un año de edad, de tamaño grande y que opera en un mercado perfectamente competitivo $(\mathrm{HHI}=0)$ tendría una probabilidad de ascenso del 52.3\% y una probabilidad de descenso de sólo 2.2\%, para una empresa hipotética promedio, es decir, pequeña y con el resto de regresores en los valores medios, la probabilidad de ascender es únicamente de 1.1\% mientras que la de descenso es de 18\%. Este resultado es interesante en la medida en que se mejora la probabilidad de ascenso con una disminución de la de descenso, lo que muestra que los factores por los cuales estas empresas fracasan están asociados a los mismos factores que ayudan a su éxito. De aquí se puede decir que una política de promoción empresarial efectiva que esté dirigida a generar cambios positivos en la posición de mercado sería una que esté orientada a empresas tipo Interrogante que sean jóvenes, grandes y que operen en mercados altamente competitivos.

\section{BIBLIOGRAFÍA}

Aláez Aller, Ricardo; Longás García, Juan Carlos; Ullibarri Arce, Miren (2001). La relación entre efectos externos y aglomeración: una aproximación a su estudio a partir de la evidencia empírica disponible. En: Revista de Estudios Regionales, n. ${ }^{\circ}$ 61, p. 151-167. 
Bechetti, Leonardo y Trovato, Giovanni (2001). The determinants of growth for small and medium sized firms. The role of the availability of external finance. [En línea] Departamental Working Papers, tor Vergata University, Roma, julio, 32p. Disponible en: <http:// art.torvergata.it/bitstream/2108/163/1/148. pdf $>$. [Consultado 10/02/2011].

Brandenburger, Adam M. y Nalebuff, Barry (1996). Coopetition: A revolution mindset that combines competition and cooperation... The game theory strategy that's changing the game of business. New York, Doubleday, 373p.

Cameron, Colin y Trivedi, Pravin (2005). Microeconometrics, methods and applications. New York, Cambridge University Press, 1058p.

Cameron, Colin y Trivedi, Pravin (2009). Microeconometrics using stata. College Station, Stata Press, 732p.

Cardona, Marleny y Cano, Carlos Andrés (2004). Ciclo de vida y localización espacial de las firmas en Colombia. En: Revista Universidad EAFIT, Vol. 41, n. 137, enero-marzo, p. 44-59.

Carpenter, Robert y Petersen, Bruce (2002). Is the growth of small firms constrained by internal finance? En: The Review of Económics and Statistics, Vol. 84, n. ${ }^{\circ}$, mayo, p. 298-309.
Church, Jeffrey y Ware, Roger (2000). Industrial organization: a strategic approach. Boston, Irwin McGrawHill, 960p.

Hausman, Jerry (1978). Specification test in econometrics. En: Econométrica, Vol. 46, n. ${ }^{\circ}$ 6, noviembre, p. $125 \overline{1-1271 .}$

Henderson, Bruce (1976). El pensamiento empresarial. 320p.

Hax, Arnoldo y Majluf, Nicolas (1984). Strategic management: an integrative perspective. Englewood Cliffs, Prentice Hall. $715 p$.

Langebaek, Andres (2008). Determinantes del crecimiento de las empresas no-financieras en Colombia: ley de gibrat y otras teorías. Borradores de Economía, n. $493,27 \mathrm{p}$.

Stalk, George y Stern, Carl (2002). Ideas sobre estrategia. Colección Management. Deusto, Barcelona, 96 p.

Tenjo, Fernando, López, Enrique y Zamudio, Nancy (2006). Determinantes de la estructura de capital de las empresas colombianas (19962002). Borradores de Economía, n. 380, marzo, $38 \mathrm{p}$. 


\section{ANEXOS}

Anexo A. Resultados pruebas de Wald

\begin{tabular}{|c|c|c|c|}
\hline EGOV & EBITDA & PKT & Afijo \\
\hline $\begin{array}{l}\operatorname{chi} 2(6)=15.03 \\
\text { Prob }>\text { chi2 }=0.0200\end{array}$ & $\begin{array}{l}\text { chi2 }(6)=13.56 \\
\text { Prob }>\text { chi2 }=0.0350\end{array}$ & $\begin{array}{l}\operatorname{chi} 2(6)=8.22 \\
\text { Prob }>\text { chi2 }=0.2224\end{array}$ & $\begin{array}{l}\text { chi2 }(6)=20.05 \\
\text { Prob }>\text { chi2 }=0.0027\end{array}$ \\
\hline Edad & Costos Hundidos & Altas Inversiones & $\mathrm{HHI}$ \\
\hline $\begin{array}{l}\operatorname{chi} 2(6)=48.16 \\
\text { Prob }>\text { chi2 }=0.0000\end{array}$ & $\begin{array}{l}\text { chi2 }(6)=84.50 \\
\text { Prob }>\text { chi2 }=0.0000\end{array}$ & $\begin{array}{l}\text { chi2 }(6)=32.23 \\
\text { Prob }>\text { chi2 }=0.0000\end{array}$ & $\begin{array}{l}\text { chi2 }(6)=17.18 \\
\text { Prob > chi2 }=0.0086\end{array}$ \\
\hline Escala & Tamaño 2 & Tamaño 3 & No significativas \\
\hline $\begin{array}{l}\text { chi2 }(6)=10.83 \\
\text { Prob }>\text { chi2 }=0.0937\end{array}$ & $\begin{array}{l}\text { chi2 }(6)=8.24 \\
\text { Prob }>\text { chi2 }=0.2213\end{array}$ & $\begin{array}{l}\text { chi2 }(6)=48.77 \\
\text { Prob }>\text { chi2 }=0.0000\end{array}$ & \begin{tabular}{|l|} 
PKT \\
Tamaño 2
\end{tabular} \\
\hline
\end{tabular}

Fuente: Elaboración propia

Anexo B. Resultados pruebas de multicolinealidad

\begin{tabular}{|l|c|c|c|c|}
\hline \multicolumn{1}{|c|}{ Variable } & VIF & SORT - VIF & Tolerancia & R2 \\
\hline EGOV & 1.03 & 1.02 & 0.9698 & 0.0302 \\
EBITDA & 1.56 & 1.25 & 0.6419 & 0.3581 \\
PKT & 1.03 & 1.01 & 0.9736 & 0.0264 \\
Afijo & 1.88 & 1.37 & 0.5309 & 0.4691 \\
Edad & 1.13 & 1.06 & 0.8856 & 0.1144 \\
Tamaño & 1.15 & 1.07 & 0.8668 & 0.1332 \\
Costos Hundidos & 1.7 & 1.3 & 0.5892 & 0.4108 \\
Altas Inversiones & 1.73 & 1.32 & 0.5781 & 0.4219 \\
HHI & 2.15 & 1.47 & 0.4652 & 0.5348 \\
Escala & 1.81 & 1.35 & 0.5526 & 0.4474 \\
\hline
\end{tabular}

Fuente: Elaboración propia

Anexo C. Resultados pruebas de Hausman

\begin{tabular}{|c|c|c|c|c|}
\hline Variables & (b) & (B) & $(6-B)$ & $\operatorname{sart}\left(d i a g\left(V_{-} b-V_{-}\right.\right.$B) $)$S.E \\
\hline EGOV & 0.001055 & 0.001031 & 0.000024 & 0.0002284 \\
\hline EBITDA & -0.005617 & -0.0055083 & -0.0001087 & 0.0004483 \\
\hline PKT & 0.001084 & 0.0010855 & -0.0000153 & 0.0001178 \\
\hline Afijo & 0.0201509 & 0.0202347 & -0.0000838 & 0.0015043 \\
\hline Edad & -0.0053889 & -0.0053733 & -0.0000156 & 0.0001259 \\
\hline Tamaño 2 & 0.2356937 & 0.2330855 & 0.0026082 & 0.0109922 \\
\hline Tamaño 3 & 0.9320779 & 0.9289216 & 0.0031563 & 0.0136558 \\
\hline Costos hundidos & -0.0380524 & -0.0381129 & 0.0000605 & - \\
\hline Altas inversiones & -0.0069661 & -0.0069728 & 0.0000673 & - \\
\hline Escala & 0.0068542 & 0.0061764 & 0.0006778 & 0.0056621 \\
\hline \multicolumn{5}{|c|}{ b es consistente bajo Ho y Ha; obtenido de un Logit multinomial } \\
\hline \multicolumn{5}{|c|}{ B es inconsistente bajo Ha, pero eficiente bajo Ho; obtenido de un Logit multinomial } \\
\hline \multicolumn{5}{|c|}{ Ho: las diferencias en los coeficientes no son sistemáticas } \\
\hline \multicolumn{5}{|c|}{$\operatorname{chi} 2(10)=(b-B)^{\prime}\left[\left(V_{-} b_{-} V_{-} B\right)^{\wedge}(-1)\right](b-B)=1.04$} \\
\hline \multicolumn{5}{|c|}{ Prob>chi2 $=0.9998$} \\
\hline
\end{tabular}

Fuente: Elaboración propia 
Anexo D. Ascenso empresas Perro por edad

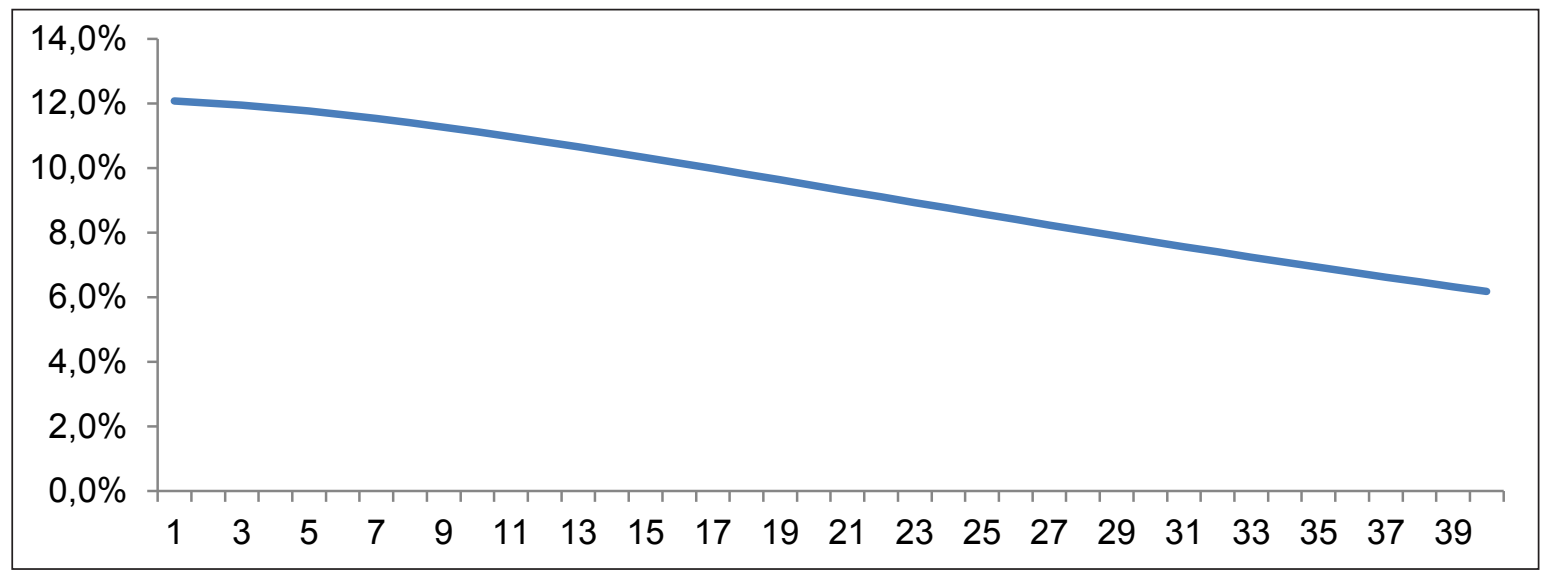

Fuente: Elaboración propia

Anexo E. Ascenso empresas Perro por tamaño

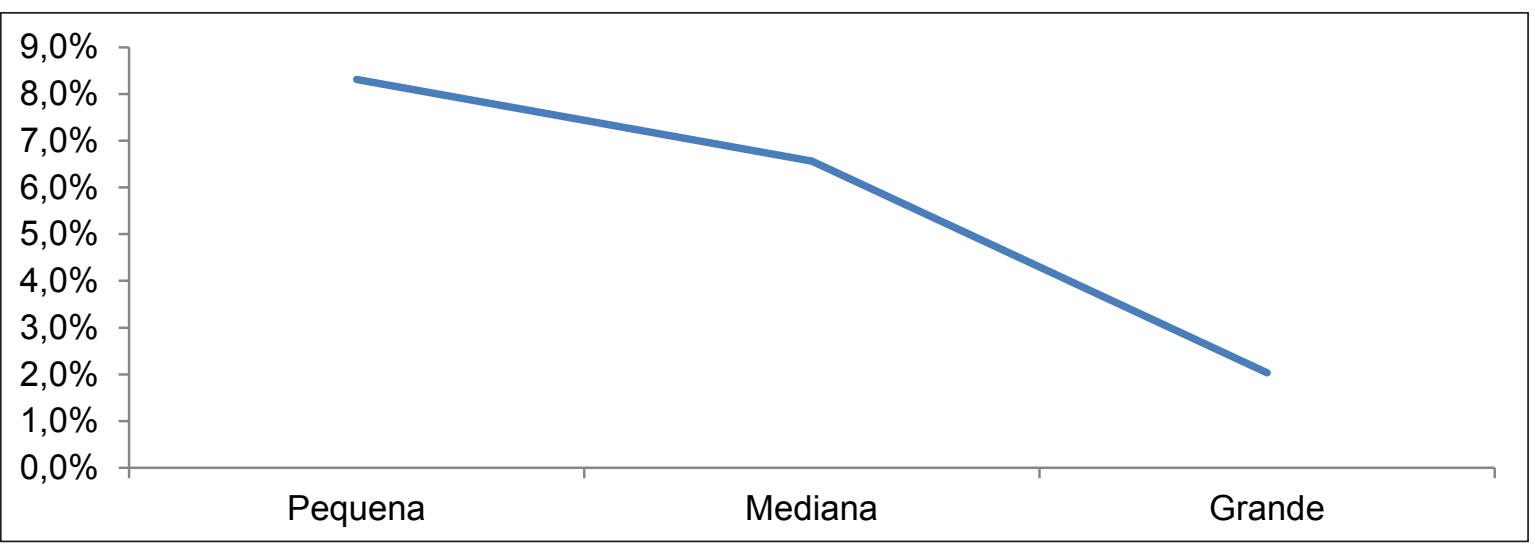

Fuente: Elaboración propia

Anexo F. Ascenso empresas Perro por HHI

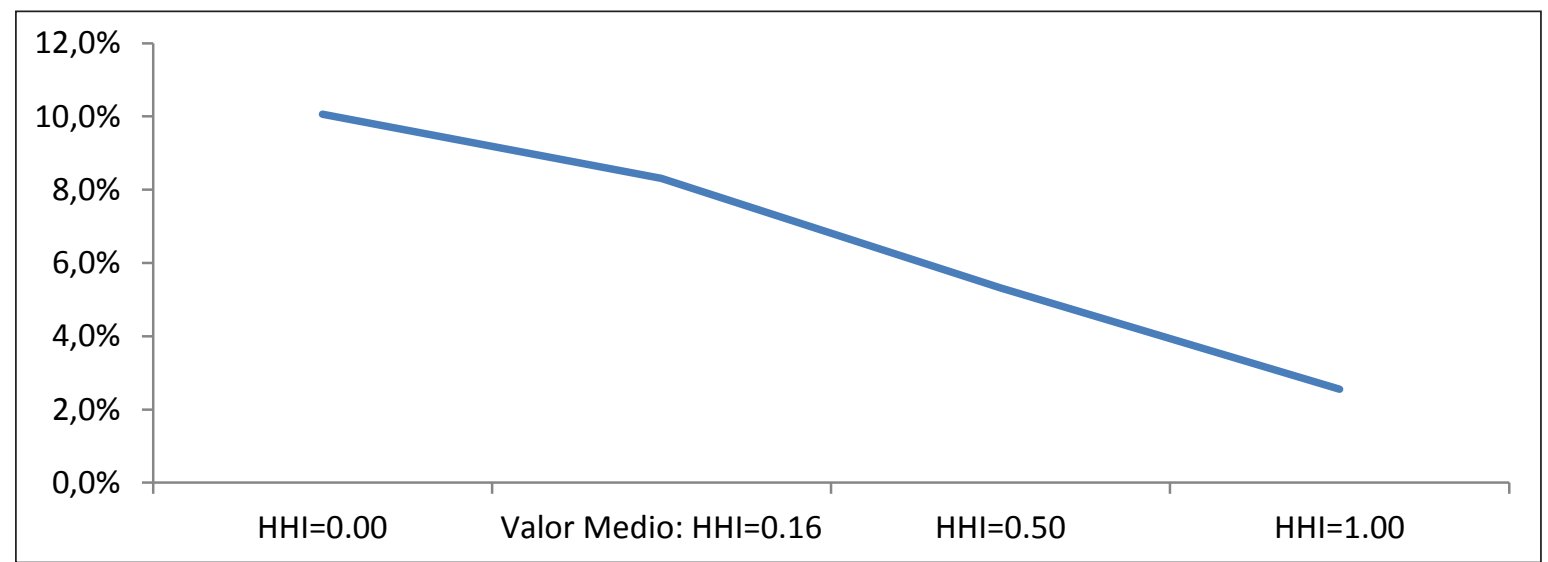

Fuente: Elaboración propia 
Anexo G. Ascenso empresas Interrogante por edad

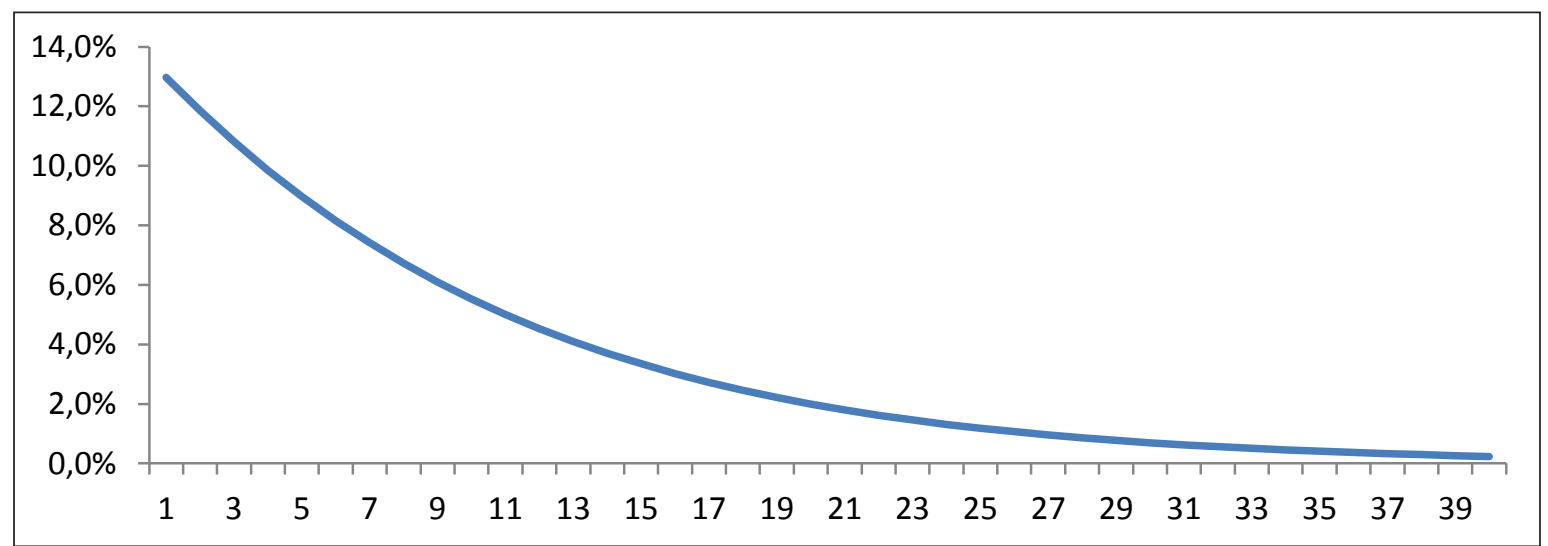

Fuente: Elaboración propia

Anexo H. Ascenso empresas Interrogante por tamaño

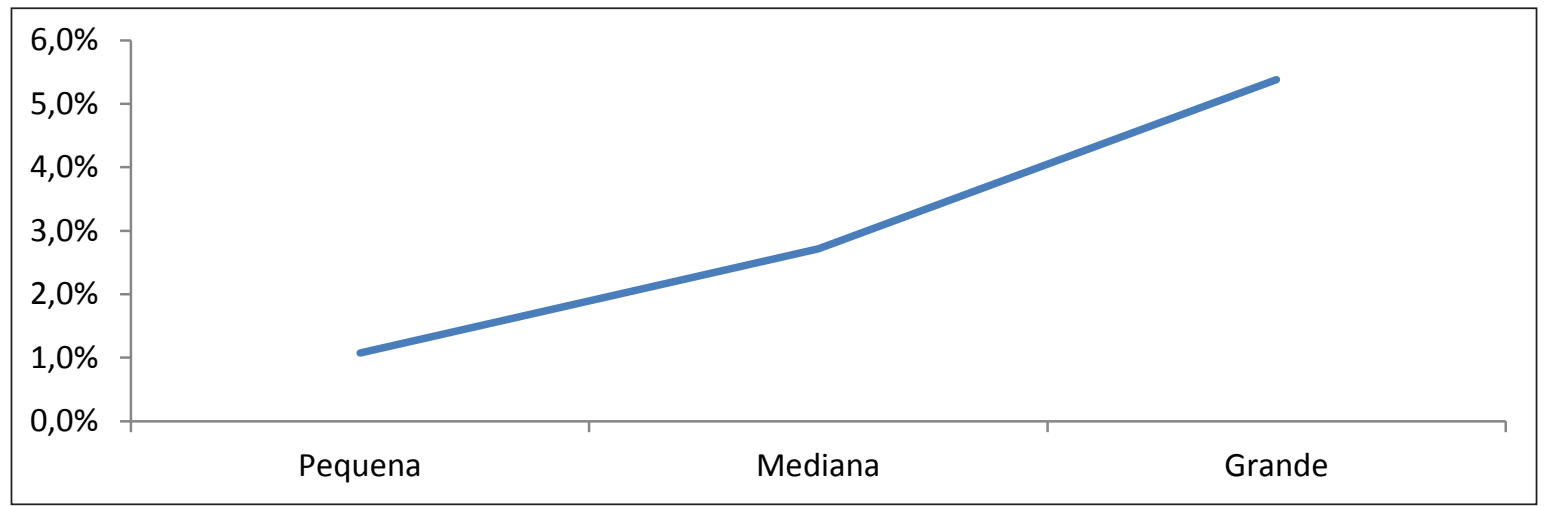

Fuente: Elaboración propia

Anexo I. Ascenso empresas Interrogante por HHI

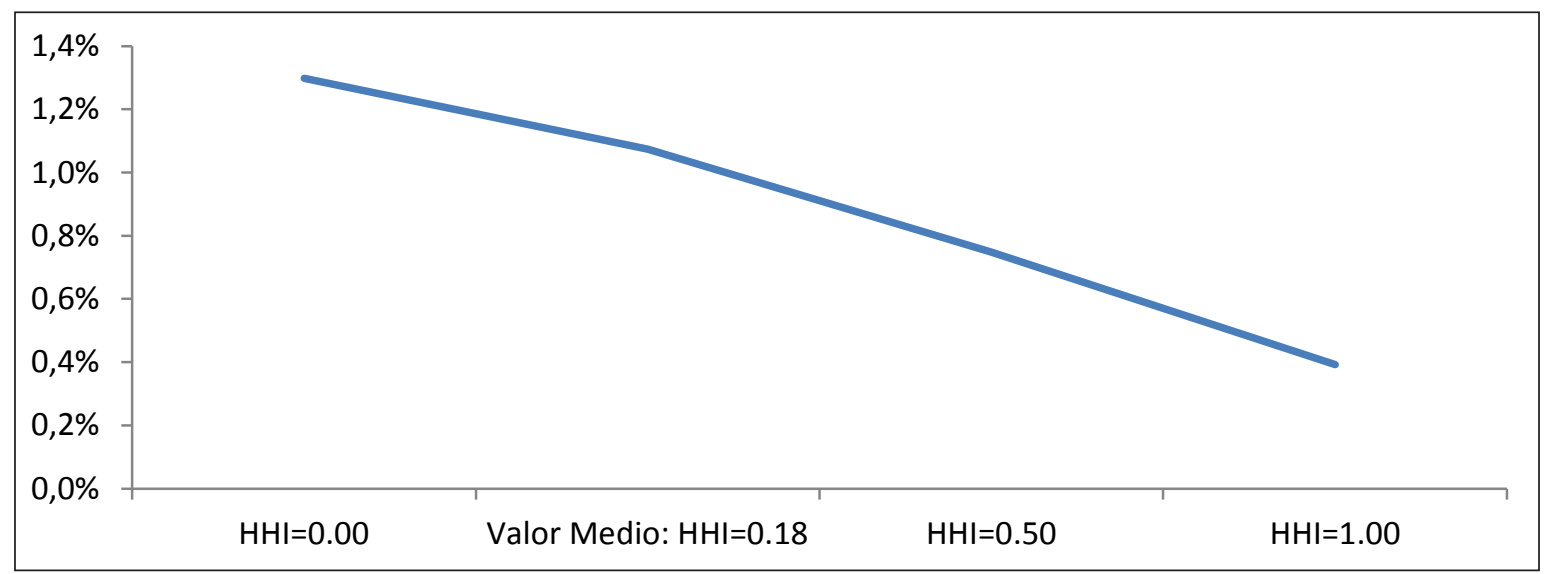

Fuente: Elaboración propia 
Anexo J. Descenso empresas Interrogante por edad

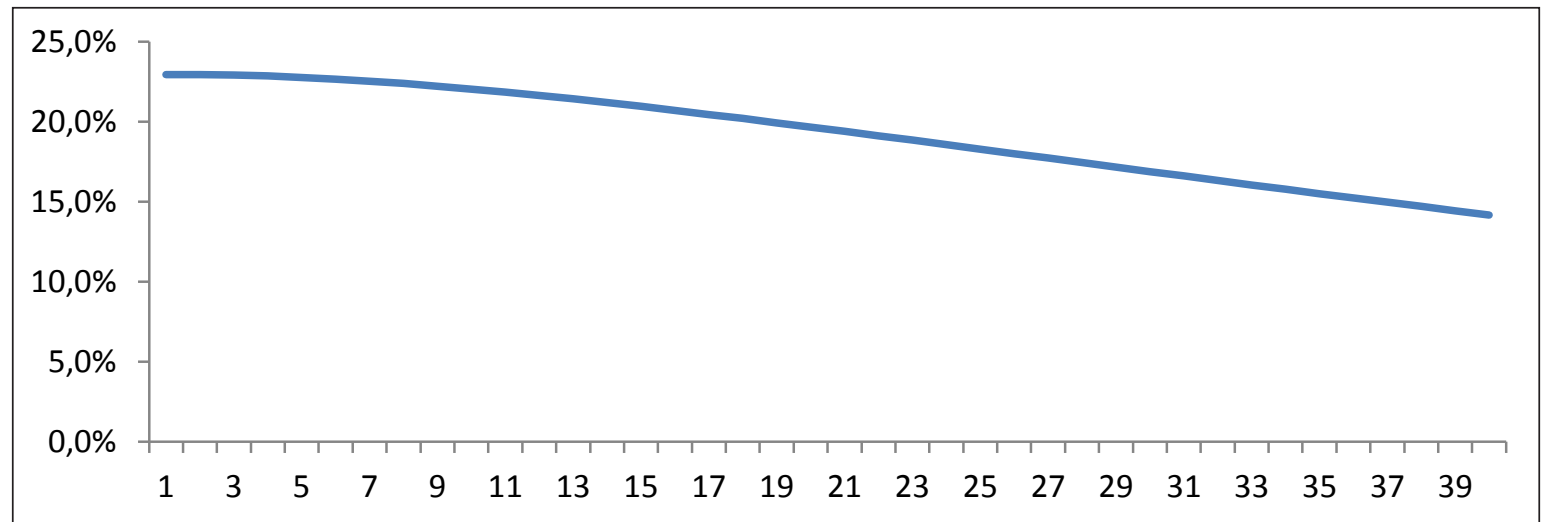

Fuente: Elaboración propia

Anexo K. Descenso empresas Interrogante por tamaño

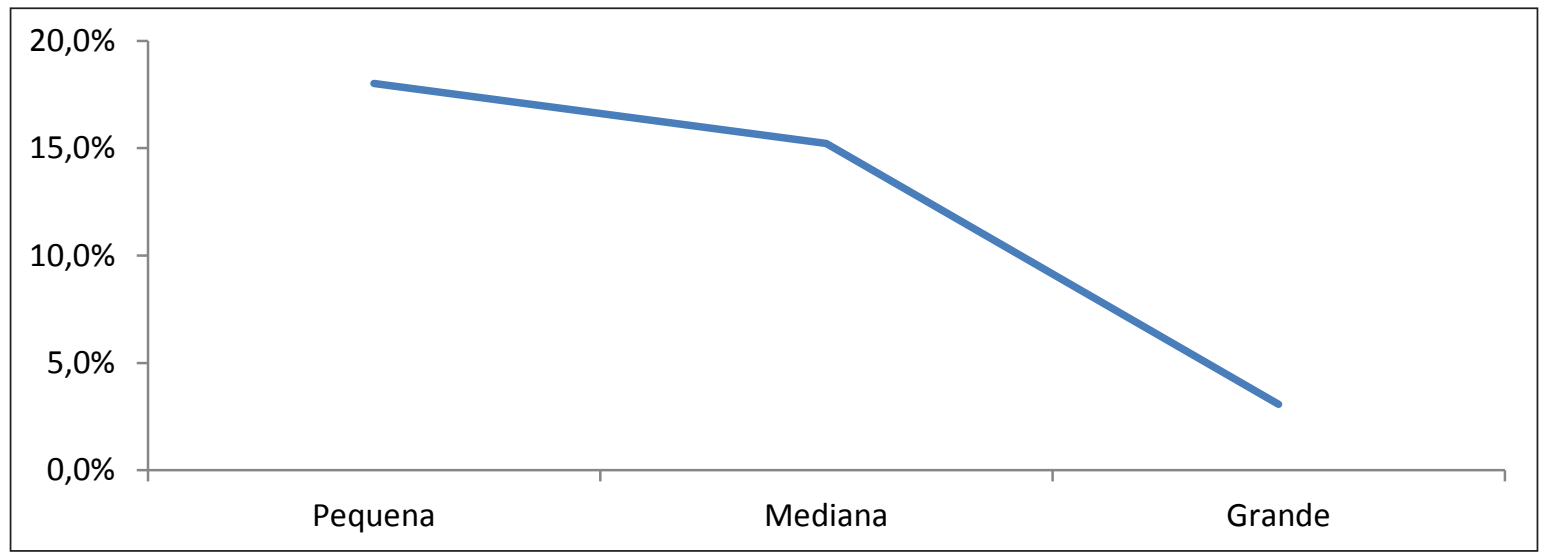

Fuente: Elaboración propia

Anexo L. Descenso empresas Interrogante por HHI

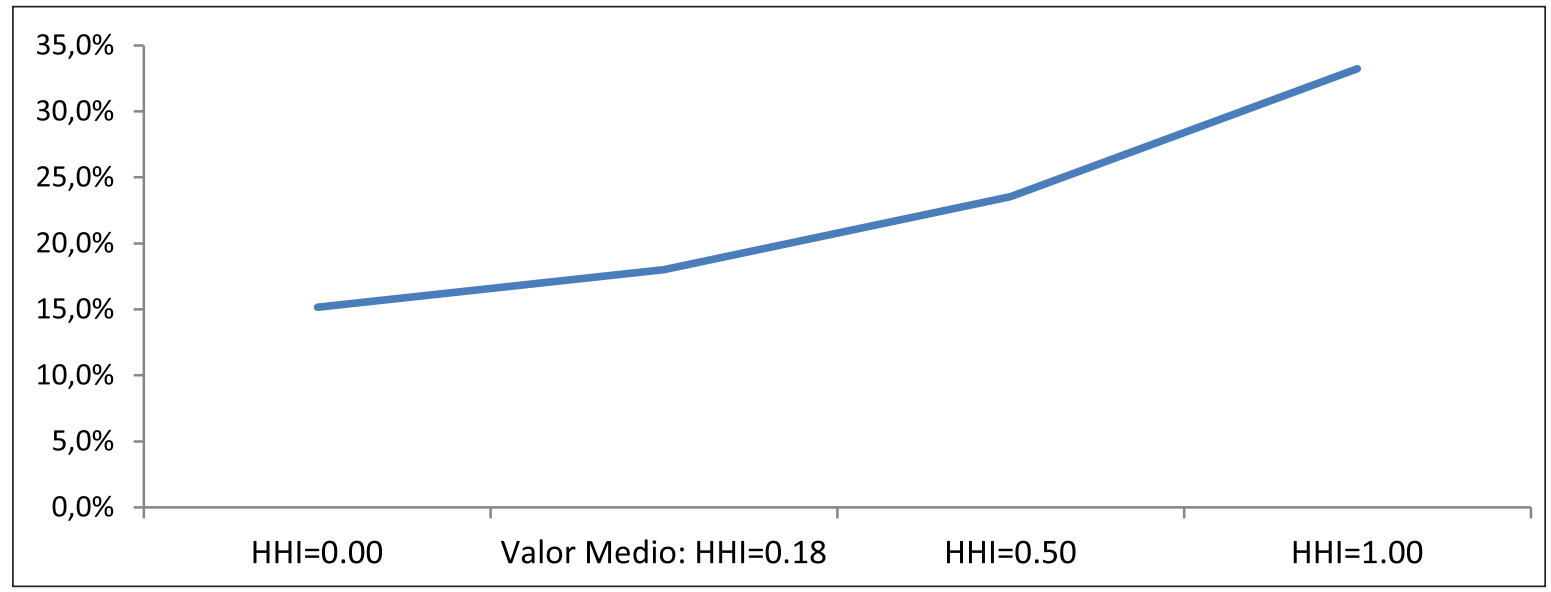

Fuente: Elaboración propia 
